\title{
Lattice QCD studies on baryon interactions in the strangeness -2 sector with physical quark masses
}

\author{
Kenji Sasaki $i^{1,2, \star}$ Sinya Aoki ${ }^{1,2,3}$, Takumi Doi ${ }^{2,4}$ Shinya Gongyo ${ }^{2,}$ Tetsuo Hatsuda ${ }^{2,4}$, \\ Yoichi lkeda ${ }^{2,5}$, Takashi Inoue ${ }^{2,6}$, Takumi Iritani $^{2}$,Noriyoshi Ishii ${ }^{2,5}$, Takaya Miyamoto ${ }^{1,2,}$ \\ for HAL QCD Collaboration \\ ${ }^{1}$ Center for Gravitational Physics, Yukawa Institute for Theoretical Physics, Kyoto University, Kyoto 606-8502, \\ Japan \\ ${ }^{2}$ Theoretical Research Division, Nishina Center, RIKEN, Wako 351-0198, Japan \\ ${ }^{3}$ Center for Computational Sciences, University of Tsukuba, Tsukuba 305-8577, Japan \\ ${ }^{4}$ iTHEMS Program and iTHES Research Group, RIKEN, Wako 351-0198, Japan \\ ${ }^{5}$ Research Center for Nuclear Physics (RCNP), Osaka University, Osaka 567-0047, Japan \\ ${ }^{6}$ Nihon University, College of Bioresource Sciences, Kanagawa 252-0880, Japan
}

\begin{abstract}
We investigate baryon-baryon $(B B)$ interactions in the strangeness $S=-2$ sector via the coupled-channel HAL QCD method which enables us to extract the scattering observables from Nambu-Bethe-Salpeter (NBS) wave function on the lattice. The simulations are performed with (almost) physical quark masses $\left(m_{\pi}=146 \mathrm{MeV}\right)$ and a huge lattice volume of $L a=8.1 \mathrm{fm}$. We discuss the fate of H-dibaryon state through the $\Lambda \Lambda$ and $N \Xi$ coupled-channel scatterings.
\end{abstract}

\section{Introduction}

The strangeness $S=-2$ sector of baryon interactions are key to explore the possibility of exotic states in two-baryon system. Numerous theoretical efforts for the strangeness $S=-2$ two-baryon system have been performed and discussed possibility of the $H$-dibaryon which is composed of six quarks (uuddss) in a spin and isospin singlet state [1,2]. Since a direct scattering experiment of hyperons is not feasible due to the short lifetimes of hyperons, their scattering data are quite limited and are insufficient to construct realistic baryon interactions. Furthermore it has been thought to be impossibly difficult to derive the $B B$ interactions by solving the dynamics of quarks and gluons from QCD because of its non-perturbative nature at low-energies.

Lattice QCD approach enables us to tackle the problem non-perturbatively via a numerical simulation. In the framework, M. Lüscher firstly proposed to relate the energy $W$ of a two-particle state in a finite box to the elastic scattering phase $\delta(W)$ in the infinite volume [3]. The relation is derived by using the asymptotic behavior of the two-particle Nambu-Bethe-Salpeter (NBS) wave function $\psi(r)$, if the range of the interaction is sufficiently smaller than the size of the box.

An alternative approach to derive the hadron interactions from lattice QCD data has been proposed in Ref. [4, 5] and has been extensively developed by the HAL QCD Collaboration [6-21], named as

${ }^{\star}$ Speaker, e-mail: kenjis@yukawa.kyoto-u.ac.jp 
HAL QCD method. In the method, we define the energy-independent and non-local potential $U\left(r, r^{\prime}\right)$ from $\psi(r)$ which obeys the Schrödinger type equation in a finite box. Using the obtained non-local potential $U\left(r, r^{\prime}\right)$ which receives only weak finite volume effect, we can simply calculate observables, such as scattering phase shifts and bound state energy if it exists, in infinite space to compare the results with experimental data.

A further advantage of the HAL QCD method is that it can be generalized straightforwardly to the case of inelastic scatterings. The coupled-channel formalism is essential for the description of the dynamics of the two-baryon systems with small energy separations which are especially realized in the strangeness -2 sector. As we shall discuss in the next section, the coupled-channel Schrödinger equation is able to be solved to obtain the potential matrix once all corresponding NBS wave functions are obtained on the lattice.

In this paper, we investigate the $B B$ interaction in the strangeness -2 sector with the $S U(3)$ breaking effects on the basis of the coupled-channel HAL QCD method developed in our previous works $[7,14]$. We also discuss the result of $\mathrm{H}$-dibaryon state at almost physical point using the $\Lambda \Lambda$ and $N \Xi$ coupled-channel potentials.

\section{Coupled-channel $B B$ potential}

We start from the normalized four-point correlation function $R$ in channel $c$ defined as

$$
R_{\mathcal{I}_{d}}^{c}(\vec{r}, t) \equiv \frac{\sum_{\vec{x}}\left\langle 0\left|B_{c_{1}}(\vec{x}+\vec{r}, t) B_{c_{2}}(\vec{x}, t) \overline{\mathcal{I}}_{d}\left(t_{0}=0\right)\right| 0\right\rangle}{\sqrt{Z_{c_{1}} Z_{c_{2}}} \exp \left[-\left(m_{c_{1}}+m_{c_{2}}\right) t\right]}=\sum_{n} \psi_{W_{n}}^{c}(\vec{r}) e^{-\Delta W_{n}^{c} t} A_{d}^{W_{n}}+\cdots,
$$

where $B_{c_{j}}(\vec{x}, t)$ is an interpolating operator for octet baryon with a channel index, $c$, and particle index, $j=1,2$, and $\sqrt{Z_{c_{j}}}$ is the corresponding wave-function renormalization factor. $\psi_{W_{n}}^{c}$ denotes the equal-time NBS wave function with the total energy $W_{n}$. An effect of source operator, $\bar{I}_{d}(0)$, emerges as $A_{d}^{W_{n}}=\left\langle W_{n}\left|\bar{I}_{d}(0)\right| 0\right\rangle$. The energy from two baryon state in the channel $c$ is denoted as $\Delta W_{n}^{c}=W_{n}-m_{c_{1}}-m_{c_{2}}$ with baryon mass $m_{c_{j}}$. The ellipses in Eq.(1) denote inelastic contributions from channels which we are not considering.

A coupled-channel potential can be obtained by using the $R$-correlator via the time-dependent Schrödinger-like equation [11] as

$$
\left(D_{t}^{c}-H_{0}^{c}\right) R_{I_{d}}^{c}(\vec{r}, t)=\int d^{3} r^{\prime} U_{e}^{c}\left(\vec{r}, \vec{r}^{\prime}\right) \Delta_{e}^{c} R_{I_{d}}^{e}\left(\vec{r}^{\prime}, t\right)
$$

where $H_{0}{ }^{c}=-\frac{\nabla^{2}}{2 \mu^{c}}$ and $\Delta^{c}{ }_{e}=\exp \left[-\left(m_{e_{1}}+m_{e_{2}}\right) t\right] / \exp \left[-\left(m_{c_{1}}+m_{c_{2}}\right) t\right]$. The operator $D_{t}^{c}$ is corresponding to the kinetic energy part of Schrödinger equation, $k_{i}^{c 2} / 2 \mu^{c}$, and described up to the second order of the time-derivative for $R$ function or, equivalently, of $\Delta W$ as

$$
D_{t}^{c} R_{I_{d}}^{c}(\vec{r}, t) \simeq-\frac{\partial}{\partial t} R_{I_{d}}^{c}(\vec{r}, t)+\frac{1}{8 \mu}\left[1+3\left(\frac{m_{c_{1}}-m_{c_{2}}}{m_{c_{1}}+m_{c_{2}}}\right)^{2}\right] \frac{\partial^{2}}{\partial t^{2}} R_{I_{d}}^{c}(\vec{r}, t)
$$

where the asymptotic momentum $k_{i}^{c}$ in the center-of-mass (CM) frame is defined via the relativistic energy as

$$
W_{n}=\sqrt{m_{c_{1}}^{2}+\left(k_{n}^{c}\right)^{2}}+\sqrt{m_{c_{2}}^{2}+\left(k_{n}^{c}\right)^{2}} .
$$

For each element of the coupled-channel potential matrix, we consider the derivative expansion to handle the non-locality of the potential as

$$
U_{e}^{c}\left(\vec{r}, \vec{r}^{\prime}\right)=\left(V_{\mathrm{LO}^{c}}^{c}(\vec{r})+V_{\mathrm{NLO}}^{c} e(\vec{r})+\cdots\right) \delta\left(\vec{r}-\vec{r}^{\prime}\right)
$$


Table 1. Baryon masses in units of MeV.

\begin{tabular}{c|r|c}
\hline \hline particle & \multicolumn{1}{|c|}{ mass } & fit range \\
\hline$N$ & $958 \pm 3$ & $16-19$ \\
$\Lambda$ & $1140 \pm 3$ & $17-20$ \\
$\Sigma$ & $1223 \pm 2$ & $17-21$ \\
$\Xi$ & $1354 \pm 1$ & $20-24$ \\
\hline \hline
\end{tabular}

where $\mathrm{N}^{n} \mathrm{LO}$ term is of $O\left(\vec{\nabla}^{n}\right)$ and its convergence has been discussed for the $N N$ case [8]. In this paper, we only consider the leading order potential of derivative expansion of the non-local potential.

\section{H-dibaryon}

In 1977 it was found out by R.L. Jaffe by using the MIT bag model that there exists a hexa-quark system where strongly attractive color-magnetic interaction is brought by one-gluon exchanges between quarks. This state which composed of [uuddss] quarks in $J^{P}=0^{+}$with $I=0$ was found to be a deeply bound state with a binding energy of about $80 \mathrm{MeV}$ and was named as the "H-dibaryon". The effect of Pauli principle among the valence quarks which is known as the one of the origin of repulsive core in nuclear force was estimated for this state and it was found that there is no the Pauli forbidden combination for spin-flavor-color space among the valence quarks. Although a large number of calculations using different models revealed the importance of the one-gluon exchange and symmetry property of quarks in the $B B$ interaction to explore a bound or resonant hexa-quark system, theoretical situation on the existence of the exotic hexa-quark state is still controversial.

From the experimental data, there are, so far, no positive signal for a presence of the H-dibaryon state for not only a deeply bound state [22] but also near the $\Lambda \Lambda$ threshold [23]. Recent analysis of scattering length for $\Lambda \Lambda$ [25] using the $\Lambda \Lambda$ correlation data in the heavy ion collision [24] suggests that there is no bound state below the $\Lambda \Lambda$ threshold.

Under such situation, the H-dibaryon search was performed by full QCD simulations on the lattice in the flavor $S U(3)$ limit [9] where the H-dibaryon is treated as the flavor singlet combination of the coupled channel two-baryon systems, $\Lambda \Lambda-N \Xi-\Sigma \Sigma$. The $B B$ interactions have been studied systematically by the HAL QCD method for several masses of the pseudo-scalar meson $m_{\mathrm{PS}}=470-$ $1170 \mathrm{MeV}$ and the H-dibayon was found for all quark masses. In order to fill the gap between the Lattice QCD results in the ideal flavor symmetric situation with heavy quark masses and experiments, the Lattice QCD study on this system at the physical point is indispensable.

\section{Lattice setup}

We employ $N_{f}=2+1$ gauge configurations which are generated with the Iwasaki gauge action at $\beta=1.82$ and nonperturbatively $O(a)$-improved Wilson quark action with $c_{S W}=1.11$ on the $L^{3} \times$ $T=96^{3} \times 96$ lattice [26]. The hopping parameters for light (ud) and strange quarks are chosen as $\left(\kappa_{u d}, \kappa_{s}\right)=(0.126117,0.124790)$ corresponding to $m_{\pi} \simeq 146 \mathrm{MeV}$ and $m_{K} \simeq 525 \mathrm{MeV}$ with $a^{-1} \simeq 2.33 \mathrm{GeV}(a \simeq 0.085 \mathrm{fm})$. This lattice setup brings about the almost physical point simulation of the $B B$ interaction on the large lattice volume of $(8.1 \mathrm{fm})^{4}$ where a finite volume effects of the $B B$ potential could be neglected.

We calculate quark propagators in consideration of a zero momentum wall source by imposing Coulomb gauge fixing at $t_{0}$ with the Dirichlet boundary condition in temporal direction at $\left|t_{D B C}-t_{0}\right|=$ 
48. We employ the unified contraction algorithm [27] for the measurements of baryon four-point correlators. The total statistics used in this report amounts to 414 configurations $\times 4$ rotations $\times 96$ wall sources. The forward and backward propagations of baryon four-point correlator are averaged and four rotated gauge configurations are used to reduce the statistical errors. The projection to the $A_{1}$-irrep of the cubic group is taken for the sink operator to project on the $\mathrm{S}$-wave in the $B B$ wave function. Jackknife prescription with the bin of the size 6 ( 69 configurations $\times 5$ trajectries) is used to estimate the statistical errors.

We emply the following interpolating operator for octet baryons,

$$
B_{\alpha}(\vec{x})=\epsilon_{a b c}\left(q_{a}^{T}(\vec{x}) C \gamma_{5} q_{b}(\vec{x})\right) q_{c \alpha}(\vec{x})
$$

with the Dirac index $\alpha$, which represents the spin of the octet baryons. The flavor structures of baryons are given as

$$
\begin{array}{ll}
N \quad: \quad p=[u d] u, \quad n=[u d] d \\
\Xi \quad: \quad \Xi^{0}=[s u] s, \quad \Xi^{-}=[s d] s \\
\Lambda \quad: \quad \Lambda=\frac{1}{\sqrt{6}}([s d] u+[u s] d-2[d u] s), \\
\Sigma \quad: \quad \Sigma^{+}=-[u s] u, \quad \Sigma^{-}=-[d s] d, \quad \Sigma^{0}=-\frac{1}{\sqrt{2}}([d s] u+[u s] d),
\end{array}
$$

where a square bracket stands for an antisymmetric combination. Focusing on the $H$-dibaryon channel, we only treat the $I=0$ combination of two-baryons defined as

$$
\begin{aligned}
& (\Lambda \Lambda)_{I=0}=\Lambda \Lambda \\
& (N \Xi)_{I=0}=\sqrt{\frac{1}{2}}\left(p \Xi^{-}-n \Xi^{0}\right), \\
& (\Sigma \Sigma)_{I=0}=\sqrt{\frac{1}{3}}\left(\Sigma^{+} \Sigma^{-}+\Sigma^{-} \Sigma^{+}-\Sigma^{0} \Sigma^{0}\right) .
\end{aligned}
$$

The baryon masses measured in this setup are listed in Tab. 1. These masses are slightly heavier than the physical values due to slightly larger quark masses at the simulation point.

\section{Results and discussions}

We now present our results of coupled-channel $B B$ potentials in strangeness $S=-2$ sector in ${ }^{1} S_{0}$ and $I=0$. The coupled-channel potentials described as $V_{i-j}$ are calculated by using the NBS wave functions at $t-t_{0}=9-12^{1}$.

In Fig. 1, the coupled-channel potentials between the $\Lambda \Lambda$ and $N \Xi$ states are summarized. We find that the $V_{\Lambda \Lambda-\Lambda \Lambda}$ potential shown in Fig. 1 (a) is stable against the change of $t$ within a range of $t=9$ to 12 , which suggests that the contaminations from inelastic excited states are suppressed well. We also find that there is a strongly repulsive core at short range region as well as a shallow attractive pocket in the $\Lambda \Lambda$ channel. This potential does not generate a bound state without considering the channel coupling effects. A weak repulsive core surrounded by an attractive pocket is observed for

\footnotetext{
${ }^{1}$ Although we confirmed that the $t$-dependence of the calculated potentials is mild, it is desirable to choose the larger $t$ in order to suppress contaminations from unconsidered inelastic states.
} 


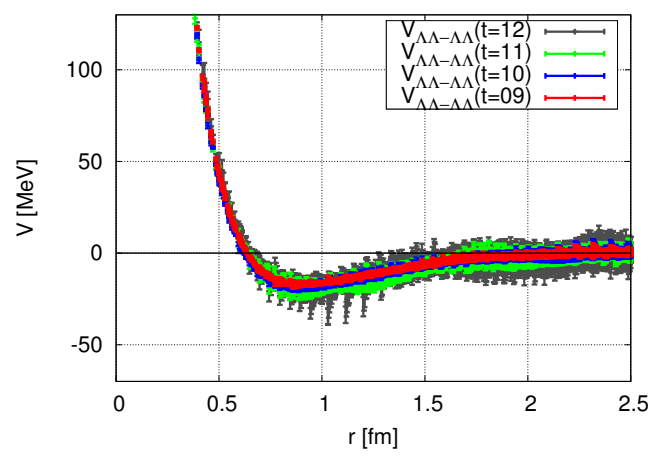

(a) $V_{\Lambda \Lambda-\Lambda \Lambda}$

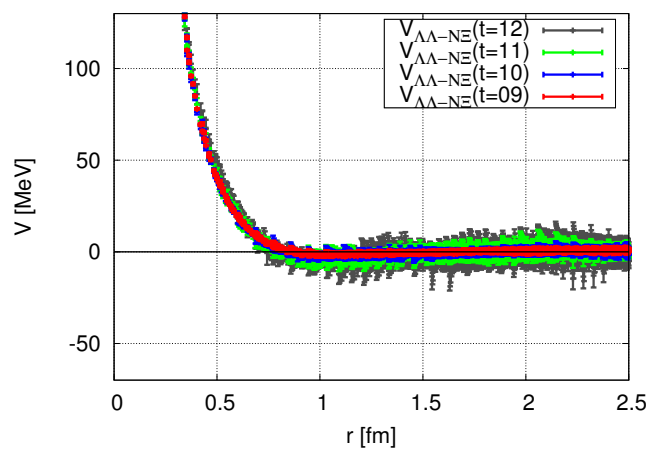

(c) $V_{\Lambda \Lambda-N \Xi}$

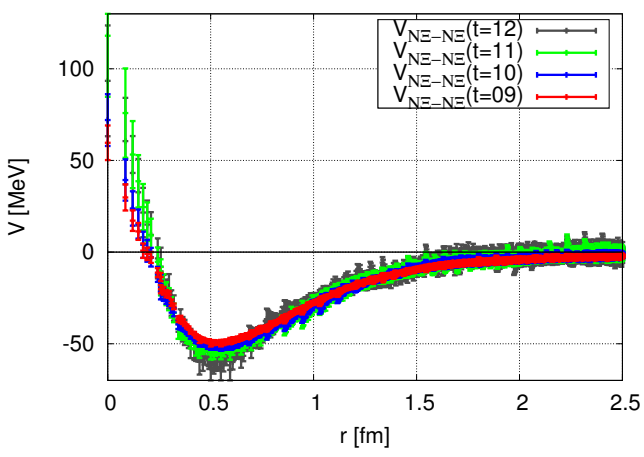

(b) $V_{N \Xi-N \Xi}$

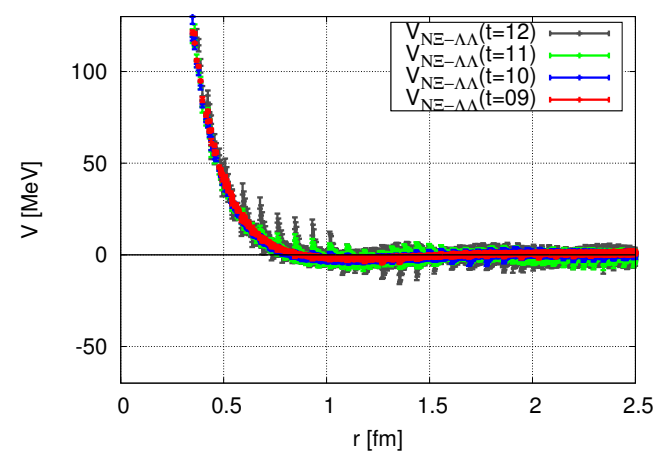

(d) $V_{N \Xi-\Lambda \Lambda}$

Figure 1. Preliminary results of $\Lambda \Lambda$ and $N \Xi$ coupled-channel potential. Figs. (a) and (b) shows the diagonal parts of potential matrix for $\Lambda \Lambda$ and $N \Xi$ channel, respectively. The transition potential of $V_{\Lambda \Lambda-N \Xi}$ and $V_{N \Xi-\Lambda \Lambda}$ are respectively given in Figs. (c) and (d).

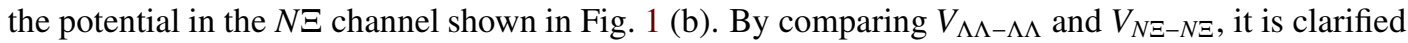
that the potential in $N \Xi$ channel is much attractive than that in $\Lambda \Lambda$ channel. Although all results of $V_{N \Xi-N \Xi}$ from $t=9$ to 12 are consistent within the statistical error, small changes against the $t$, which means growing repulsion and deepening attractive pocket, are observed unlike the case of the $\Lambda \Lambda$ potential.

The off-diagonal parts of potential matrix are shown Fig. 1 (c) and (d). By comparing these two figures, we find that both parts of transition potentials are consistent within the error bars, and besides, these potentials have no dependence of $t$. This observation indicates that the unitarity of $S$-matrix in this system is preserved. What has to be noticed is that the transition potential is very weak for $r \geqq 1 \mathrm{fm}$ region. It is inferred that the coupling between the $\Lambda \Lambda$ and $N \Xi$ channels occurs only in the short range region and coupled-channel effects are very small in the low-energy scatterings.

In Fig. 2, the phase shifts of $\Lambda \Lambda$ and $N \Xi$ channels and inelasticity of scattering are shown. From Fig. 2(a), we find that, except for the result at $t=12$, the $\Lambda \Lambda$ phase shift cuts through $90^{\circ}$ just below the $N \Xi$ threshold, which is a definition of a resonance state. The same plot above the $N \Xi$ threshold energy with shifting $180^{\circ}$ downward for the results at $t=9-11$ is drawn in Fig. 2 (a'). It indicates that there is only a small effect of the existence of sharp resonance to the $\Lambda \Lambda$ scattering phase shift above the $N \Xi$ threshold. On the other hand, according to Fig. 2 (b), we observe that the drastic change 


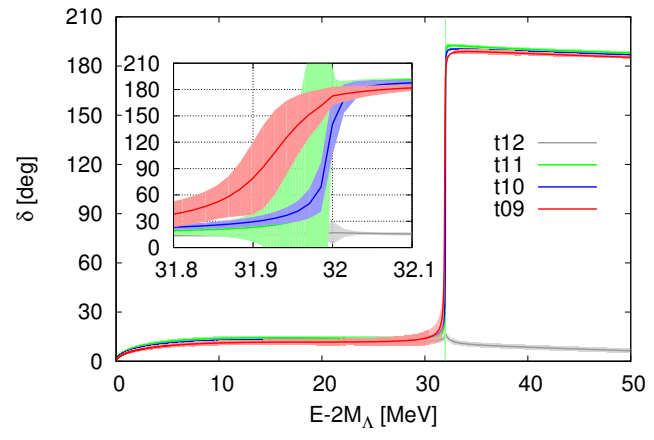

(a) $\Lambda \Lambda$ phase shift

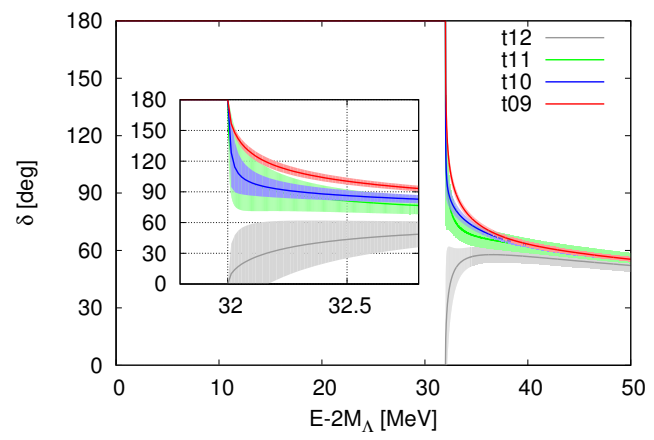

(b) $N \Xi$ phase shift.

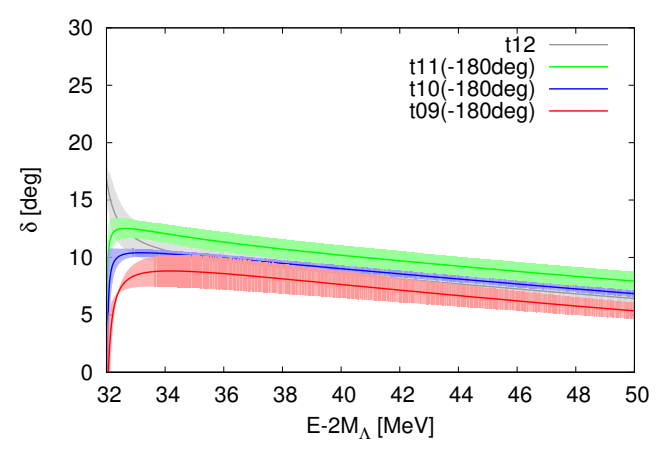

(a') $\Lambda \Lambda$ phase shift above the $N \Xi$ threshold.

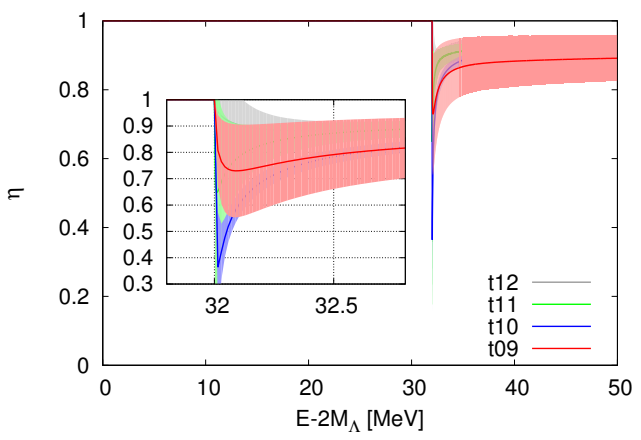

(c) Inelasticity.

Figure 2. Preliminary results of $\Lambda \Lambda$ and $N \Xi$ scattering phase shifts. The $\Lambda \Lambda$ phase shift is given in Fig. 2(a) and the same plot above the $N \Xi$ threshold energy with shifting $180^{\circ}$ downward for the results at $t=9-11$ is given in Fig. 2 (a'). The $N \Xi$ phase shift and the inelasticity of scattering is given in Fig. 2(b) and (c), respectively.

of $N \Xi$ phase shift at $t=12$ from the others shows that the resonance pole found at $t=9-11$ turns to be the virtual pole in $N \Xi$ state but such difference is seen in only a few $\mathrm{MeV}$ above the $N \Xi$ threshold. Following the weak transition potential between $\Lambda \Lambda$ and $N \Xi$ channels, the resonance which can be seen in the result at $t=9-11$ in Fig. 2 (a) would be very sharp and the inelasticity of scattering is small as seen in Fig. 2 (c). The smallness of coupled-channel effects is expected to lead an important result for the lifetime of $\Xi$-hypernuciei because it suppresses the strong decay of $N \Xi$ into $\Lambda \Lambda$.

As a consequence of Fig. 2, it is difficult to say whether the resonance state in $\Lambda \Lambda$ scattering survives in nature or not. However, we clarify that the close investigation of the strength of $N \Xi$ potential affects the fate of $\mathrm{H}$-dibaryon in nature.

\section{Conclusions}

We have investigated $S=-2 B B$ interactions from lattice QCD employing $N_{f}=2+1$ gauge configurations with $(96 a)^{4}$ and $a \simeq 0.085 \mathrm{fm}$ lattice, where $m_{\pi} \simeq 146 \mathrm{MeV}$ and $m_{K} \simeq 525 \mathrm{MeV}$. Baryon potentials have been calculated by the coupled-channel HAL QCD method with considerations of not only spacial but also temporal correlations of baryon four-point correlators. We have shown prelimi- 
nary results of coupled-channel potential of $\Lambda \Lambda-N \Xi$ system with $J=0$ and $I=0$ and their scattering observables.

The coupled-channel potentials show that they are stable against the change of $t$ for $V_{\Lambda \Lambda-\Lambda \Lambda}$, $V_{\Lambda \Lambda-N \Xi}$ and $V_{N \Xi-\Lambda \Lambda}$. The strength of off-diagonal potentials, $V_{\Lambda \Lambda-N \Xi}$ and $V_{N \Xi-\Lambda \Lambda}$, indicate that the coupling between $\Lambda \Lambda$ and $N \Xi$ states is weak. For the case of $N \Xi$ potential, we observe a mild dependence of $t$ for both repulsive core and attractive pocket as seen in Fig. 1 (b). Small fluctuation of the $N \Xi$ potential against $t$ yields a drastic change of $\Lambda \Lambda$ and $N \Xi$ phase shifts at only around the $N \Xi$ threshold. This can be explained by the small change of pole position in the scattering $S$-martix in the complex energy plane which is generated mainly by the strong attraction in $N \Xi$ channel.

As a conclusion, there is no doubt that the strength of $N \Xi$ attraction is key to clarify the fate of $\mathrm{H}$-dibaryon in nature. The momentum correlation of the $p \Xi^{-}$system from relativistic heavy ion collisions will give useful constraint on the $N \Xi$ interaction [28]. Such measurements will be performed at RHIC and LHC and the experimental data will be compared to the theoretical analysis to determine the strength of $N \Xi$ attraction.

\section{Acknowledgments}

We thank members of PACS Collaboration for the gauge configuration generation. The lattice QCD calculations have been performed on the K computer at RIKEN, AICS (hp120281, hp130023, hp140209, hp150223, hp150262, hp160211, hp170230), HOKUSAI FX100 computer at RIKEN, Wako (G15023, G16030, G17002) and HA-PACS at University of Tsukuba (14a-20, 15a-30). T.D. and T.H. are supported in part by RIKEN iTHES Project and iTHEMS Program. We thank ILDG/JLDG [29] which serves as an essential infrastructure in this study. This work is supported in part by MEXT as SPIRE (Strategic Program for Innovative REsearch) Field 5 project, "Priority Issue on Post-K computer" (Elucidation of the Fundamental Laws and Evolution of the Universe) and Joint Institute for Computational Fundamental Science (JICFuS).

\section{References}

[1] R. L. Jaffe, Phys. Rev. Lett. 38 (1977) 195 [Erratum-ibid. 38 (1977) 617].

[2] T. Sakai, K. Shimizu and K. Yazaki, Prog. Theor. Phys. Suppl. 137 (2000) 121.

[3] M. Lüscher, Nucl. Phys. B 354 (1991) 531.

[4] N. Ishii, S. Aoki and T. Hatsuda, Phys. Rev. Lett. 99 (2007) 022001.

[5] S. Aoki et al. [HAL QCD Collaboration], Prog. Theor. Exp. Phys. (2012) 01A105.

[6] H. Nemura, N. Ishii, S. Aoki and T. Hatsuda, Phys. Lett. B 673 (2009) 136.

[7] S. Aoki et al. [HAL QCD Collaboration], Proc. Jpn. Acad., Ser. B, 87 (2011) 509.

[8] K. Murano, N. Ishii, S. Aoki and T. Hatsuda, Prog. Theor. Phys. 125 (2011) 1225.

[9] T. Inoue et al. [HAL QCD Collaboration], Nucl. Phys. A 881 (2012) 28.

[10] T. Doi et al. [HAL QCD Collaboration], Prog. Theor. Phys. 127 (2012) 723.

[11] N. Ishii et al. [HAL QCD Collaboration], Phys. Lett. B 712 (2012) 437.

[12] K. Murano et al. [HAL QCD Collaboration], Phys. Lett. B 735 (2014) 19.

[13] Y. Ikeda et al. [HAL QCD Collaboration], Phys. Lett. B 729 (2014) 85.

[14] K. Sasaki et al. [HAL QCD Collaboration], Prog. Theor. Exp. Phys. (2015) 113B01.

[15] F. Etminan et al. [HAL QCD Collaboration], Nucl. Phys. A 928 (2014) 89.

[16] Y. Ikeda et al. [HAL QCD Collaboration], Phys. Rev. Lett. 117 (2016) no.24, 242001.

[17] M. Yamada et al. [HAL QCD Collaboration], PTEP 2015 (2015) no.7, $071 \mathrm{~B} 01$. 
[18] T. Miyamoto [HAL QCD Collaboration], PoS LATTICE 2015 (2016) 090.

[19] T. Iritani et al. [HAL QCD Collaboration], Phys. Rev. D 96 (2017) no.3, 034521.

[20] S. Gongyo et al. [HAL QCD Collaboration], arXiv:1709.00654 [hep-lat].

[21] D. Kawai et al. [HAL QCD Collaboration], arXiv:1711.01883 [hep-lat].

[22] H. Takahashi et al., Phys. Rev. Lett. 87 (2001) 212502.

[23] B. H. Kim et al. [Belle Collaboration], Phys. Rev. Lett. 110 (2013) no.22, 222002.

[24] L. Adamczyk et al. [STAR Collaboration], Phys. Rev. Lett. 114 (2015) no.2, 022301.

[25] K. Morita, T. Furumoto and A. Ohnishi, Phys. Rev. C 91 (2015) no.2, 024916.

[26] K.-I. Ishikawa et al. [PACS Collaboration], PoS LATTICE 2015 (2016) 075.

[27] T. Doi and M. G. Endres, Comput. Phys. Commun. 184 (2013) 117.

[28] T. Hatsuda, K. Morita, A. Ohnishi and K. Sasaki, Nucl. Phys. A 967 (2017) 856.

[29] See "http://www.lqcd.org/ildg" and "http://www.jldg.org" 